\title{
ELECTRÓNICA DE POTENCIA: APLICACIÓN EN FUENTES DE ENERGÍA RENOVABLES
}

\section{POWER ELECTRONICS: APPLICATIONS IN RENEWABLE ENERGY SOURCES EDP Y ENERGIA RENOVABLE}

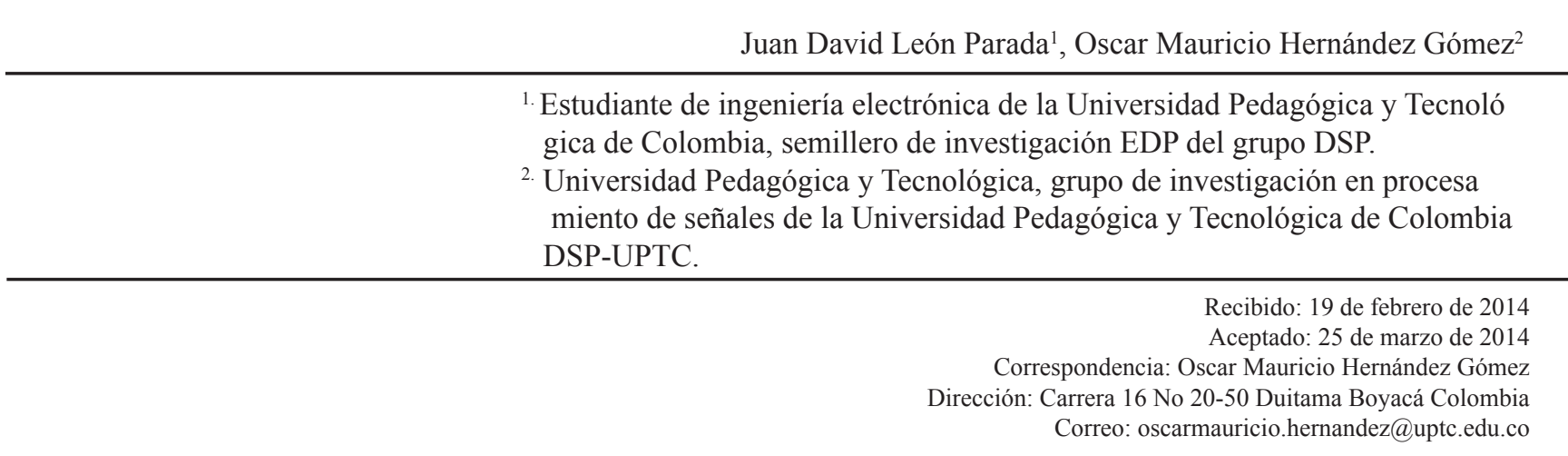

\section{RESUMEN}

La demanda de energía eléctrica ha aumentado considerablemente en los últimos años, pero el uso de fuentes de energía no renovables tienen un negativo impacto ambiental, lo que hace necesario que se desarrollen nuevas tecnologías para el uso de fuentes de energía renovables y limpias como la energía eólica. Este Artículo es una revisión de las más recientes tecnologías desarrolladas para el manejo de energías provenientes de fuentes renovables y el uso de sistemas electrónicos de potencia en ellas.

Palabras clave—Sistemas de potencia, conversión de energía, generadores, control.

\begin{abstract}
IIn recent years the use of electricity has increased considerably, but the use of non-renewable energy sources have a negative environmental impact, making it necessary to use new technologies for renewable energy and clean energy like wind energy. This article is a review of the most recent technologies developed for the management of energy from renewable sources and the use of power electronic systems.
\end{abstract}

Keywords - Power systems, energy conversion, generators, control. 


\section{INTRODUCTION}

En los sistemas de producción de energía actuales se combina tanto energía proveniente de fuentes no renovables (combustibles fósiles) como energía proveniente de fuentes renovables (eólica, Celdas fotovoltaicas, máquinas de vapor, entre otras). La energía proveniente de fuentes no renovables presenta ciertas desventajas que se deben solucionar para ayudar a suplir la demanda de energía que ha venido aumentando en los últimos años (1). Las principales desventajas de dichos sistemas son el elevado costo de muchos de los dispositivos empleados para la conversión de energía y la no controlabilidad haciendo referencia al manejo de energías provenientes de fuentes que cambian constantemente al depender de factores estacionarios, por ejemplo, la energía solar depende mucho de la temporada puesto que la cantidad de radiación solar varia a lo largo del año en muchas zonas del planeta, esta desventaja es un gran problema cuando la demanda de energía aumenta (2).

En el campo de fuentes de energía renovables, la conversión de energía eólica en energía eléctrica es de las fuentes más utilizadas, esta requiere de sofisticados sistemas mecánicos para aprovechar mejor la energía. Esta forma de producción de energía empezó a utilizarse en la década de los 80 s, se producían inicialmente unas cuantas decenas de $\mathrm{kW}$, dichos sistemas utilizaban un generador por inducción electromagnética de jaula de ardilla que se conectaba directamente a la red, por eso los cambios de intensidad del viento, uno de los principales problemas ya mencionados, producían inconsistencias, esto es, no existía control en la potencia activa y reactiva generada, dichas inconsistencias se traducen en armónicos que eran transferidos directamente a la red, lo cual era poco práctico, este problema se convirtió en un factor importante al crecer la demanda de energía, lo cual hizo necesario implementar dispositivos electrónicos de potencia para solucionarlo (3).

\section{SISTEMAS ELECTRÓNICOS DE POTENCIA MODERNOS}

El esquema de los sistemas de potencia más utilizados se muestra en la Figura 1, este esquema es aplicable tanto en sistemas de generación, como en aplicaciones de consumo en los que interviene la red eléctrica. Se observa que el sistema de conversión de energía es la interface entre la red y la carga/generador, también se aprecia un bloque de control, que es el encargado de ajustar los parámetros de rendimiento del dispositivo de potencia de acuerdo a una referencia y a la dirección del flujo de energía.

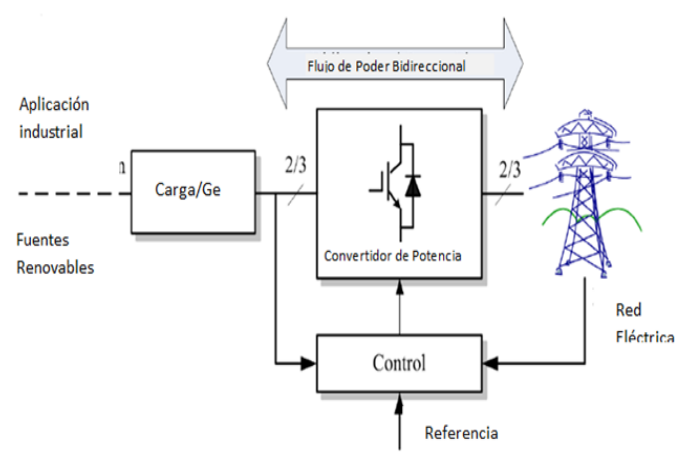

Figura 1 Esquema típico de sistemas de potencia carga/ generador en los que interviene la red eléctrica.

Son 3 aspectos que debe tener en cuenta un diseñador de dispositivos de conversión de energía basándose en el esquema típico mostrado en la Figura 1 (1)(2):

Confiabilidad.

Eficiencia.

Costo.

\section{CONVERSIÓN DE ENERGÍA}

Para la conversión de la energía normalmente se aplican técnicas que dependen de la variación de un parámetro; en la energía eólica el parámetro más importante es la velocidad puesto que la conversión de energía eólica a energía eléctrica usando generadores se ve afectada por variaciones en la velocidad de los rotores. Existen 2 tipos de conversión de energía de acuerdo al parámetro velocidad, conversión por velocidad fija, o conversión por velocidad variable, en la primera no es necesaria la implementación de electrónica de potencia, sin embargo hace uso de elementos pasivos para compensar la potencia reactiva, en la segunda se implementan dispositivos de control y potencia para variar los parámetros del generador generalmente el flujo de los polos.

En sistemas de generación de energía solar es siempre necesaria la implementación de dispositivos electrónicos de potencia de mediana complejidad, ya que para ese tipo de fuente de energía se obtiene un valor DC que cambia de acuerdo a la variación en la radiación solar, al ser la radiación solar una variable. 


\section{Sistemas eólicos con velocidad fija}

En los sistemas con velocidad fija se implementan distintas estrategias de control para mantener la velocidad en rangos deseados, dichas estrategias son el control de la inclinación de las aspas, el control de la posición y el control activo de posición.

El sistema de control de inclinación moverá las aspas a una posición angular determinada para hacer que la velocidad de giro sea constante, esto lo logra obteniendo continuamente datos de dirección y velocidad del viento.

El control por posición de las aspas logra mantener una velocidad fija modificando la posición de toda la parte rotativa de la turbina de acuerdo a la dirección del viento.

El control activo de posición combina las 2 anteriores técnicas de control de velocidad, además de posicionar la parte rotativa, puede también modificar la inclinación angular de las aspas, logrando un cambio más rápido en la velocidad (4).

En la Figura 2 se observa que luego de la etapa de conversión de energía mecánica, se utilizan bloques de compensación de potencia reactiva antes de realizar la conexión con la red, dichos bloques se componen de bancos de capacitores que se alternan continuamente de acuerdo con la variación de la producción o consumo de energía (4),(5).

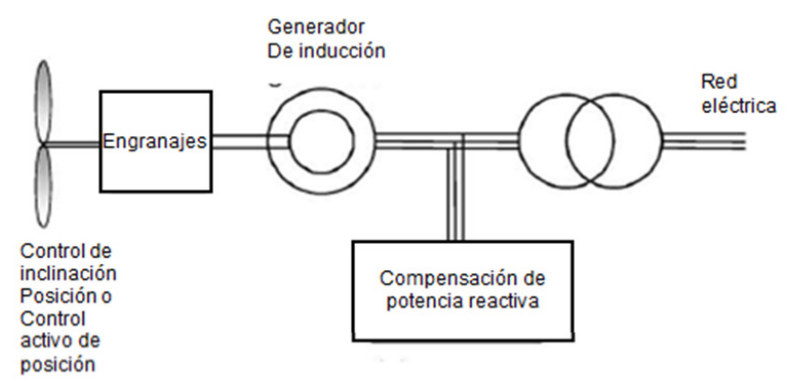

Figura 2 Sistemas de generación con velocidad fija.

Algunas topologías incorporan también bloques de compensación de arranque que suavizan los sobre picos que pueden producirse al realizar la conexión con la red, los cuales se componen de convertidores $\mathrm{AC} /$ AC, esta topología se muestra en la Figura 3 (6).

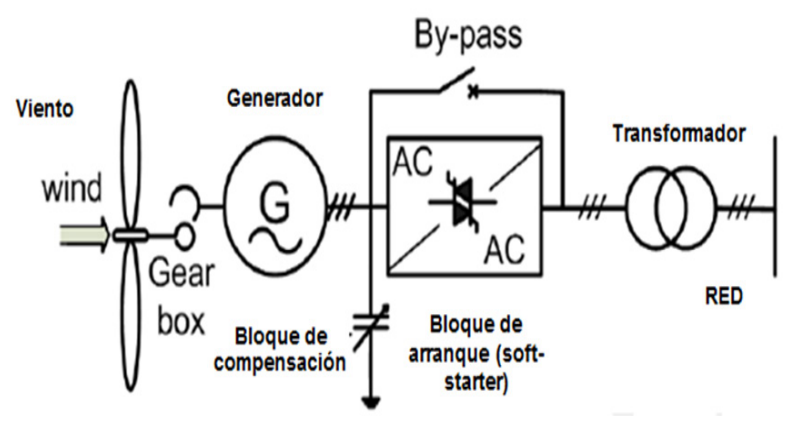

Figura 3 Sistemas de generación con velocidad fija que incorpora un bloque de arranque.

\section{Sistemas eólicos con velocidad variable}

Dentro de la categoría de sistemas de generación eólicos con velocidad variable tenemos 2 subcategorías. Los sistemas "partially rated" y los sistemas con convertidores a full escala.

En la Figura 4 se aprecia un sistema que utiliza dispositivos de potencia de baja carga (partially rated), el cual incorpora una resistencia en el rotor que es controlada para tener una generación de energía constante ante variaciones de velocidad del rotor, al igual que el modelo anterior, este diseño presenta un bloque de compensación de potencia reactiva antes de transferir la energía a la red (7).

En la Figura 5 se presenta otra alternativa usando dispositivos de potencia, consiste en una red realimentada que usa conversores conectados al rotor, la idea es regular la potencia de salida sensándola y calculando el error con respecto a una referencia, la corrección se hace por medio de otro conversor, en este caso dc/ac el cual realiza la conexión eléctrica con el rotor.

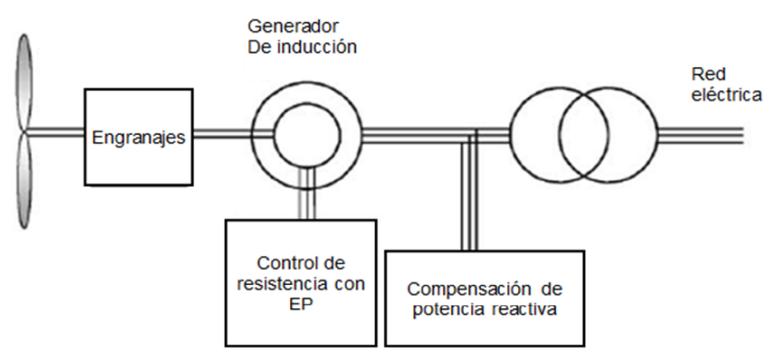

Figura 4 Sistema con velocidad variable y control de parámetros del generador.

Dentro de los sistemas "partially rated" se tiene el generador de inducción doblemente realimentado (DFIG: Doubly Fed Induction Generator) mostrado en la Figura 6, se aprecia que el estator está conec- 


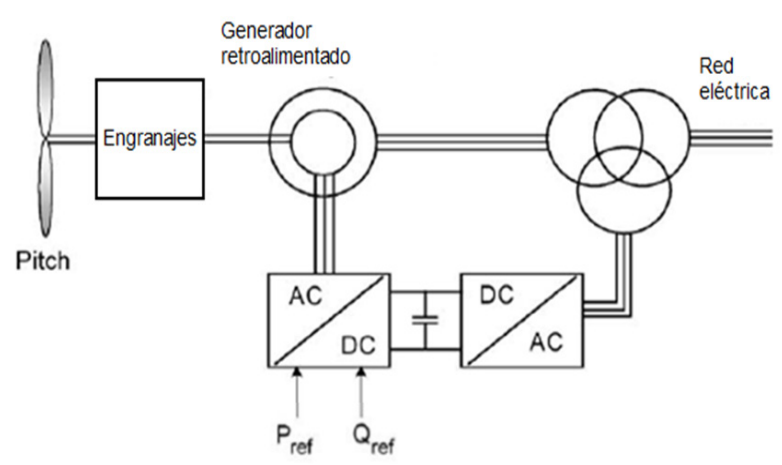

Figura 5 Sistema con velocidad variable reatroalimentado.

tado directamente a la red mientras que el bobinado del rotor está conectado a un convertidor back to back usando anillos, esto para hacer que el generador opere a una velocidad variable limitada.

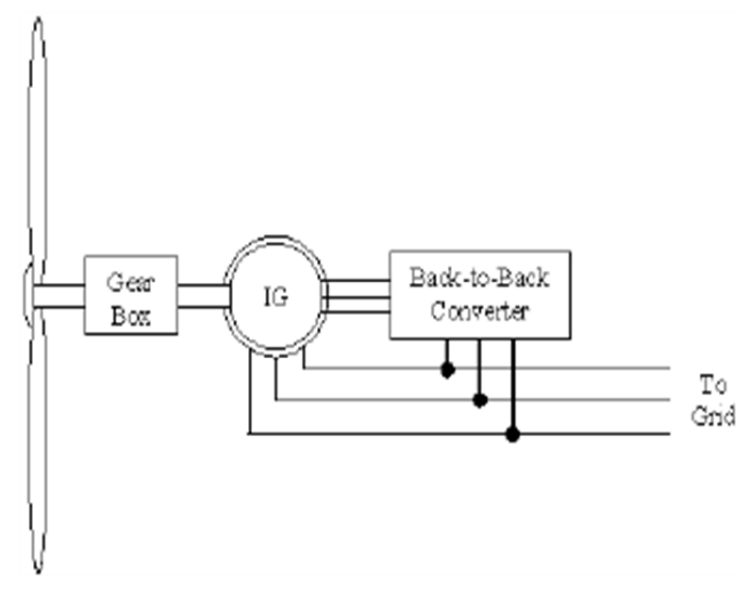

Figura 6 Sistema DFIG

Con este convertidor back to back es posible controlar la velocidad o el torque del generador y también la potencia reactiva en el lado del estator.

El esquema detallado del convertidor back to back se muestra en la Figura 7, en ella se aprecia que el convertidor se compone de IGBTs conectados como un rectificador trifásico de onda completa controlado por PWM, esta topología se usa para convertir una forma de onda senoidal a un nivel DC, eliminar fluctuaciones de baja frecuencia con el banco de capacitancias y nuevamente obtener una forma de onda de corriente senoidal con una frecuencia estable (8).

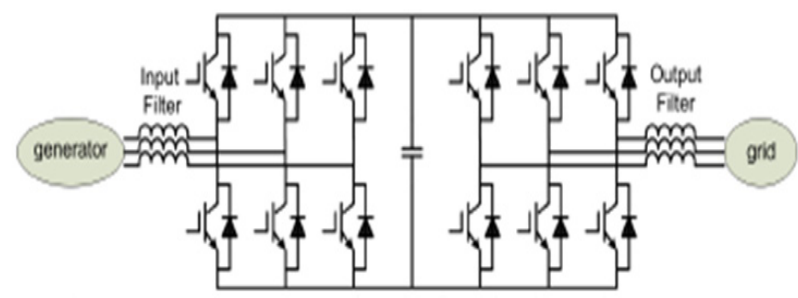

Figura 7 Esquema convertidor Back to Back

El convertidor back to back también es usado en los dispositivos con convertidores a full escala, la topología de estos sistemas se muestra en la Figura 8, dicho convertidor se encarga directamente de controlar la calidad de la energía eléctrica introducida a la red, de acuerdo a la normatividad.

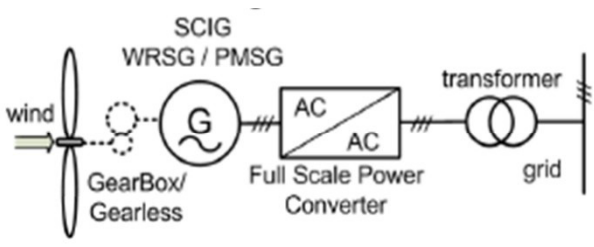

Figura 8 Sistema de velocidad Variable con Convertidor full escala

Sistemas de energía solar

Son sistemas que aprovechan la radiación proveniente del sol, el método para convertir la energía se basa en el efecto fotoeléctrico, utiliza celdas solares distribuidas a lo largo de una superficie expuesta al sol, esta forma de generación de energía, al igual que la energía eólica, depende de factores estacionarios, en este caso la radiación solar que varía a lo largo del año dependiendo de la estación en las zonas templadas del planeta, dichas variaciones son un problema menor ya que la energía producida por estos sistemas puede ser fácilmente almacenada en bancos de baterías, siguiendo la topología básica mostrada en la Figura $9(9)$.

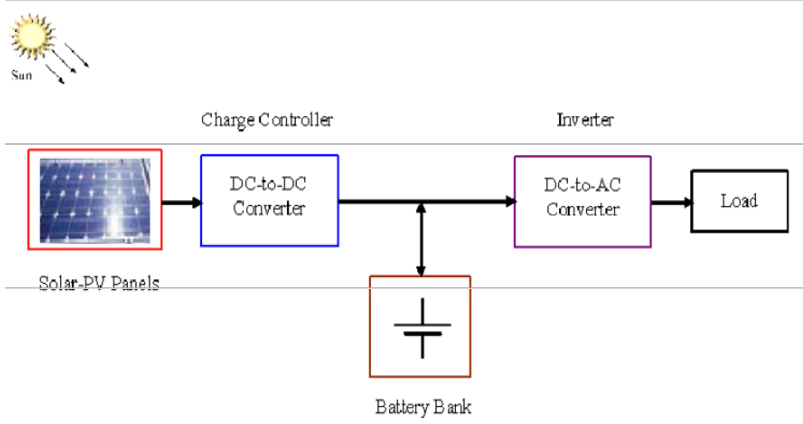

Figura 9 Configuración básica para un sistema de generación de energía solar. 
Estos sistemas usan dos tipos de dispositivos de potencia para su funcionamiento, primero un convertidor DC/ DC para regular el flujo de energía que va hacia los bancos de baterías, el segundo es un convertidor DC/AC el cual acopla el sistema con la red eléctrica o con cargas de corriente alterna.

\section{CONCLUSIONES}

Se presentó una revisión acerca de los uso de la electrónica de potencia asociada a fuentes de energía renovables, particularmente los convertidores usados para el manejo de la energía, indicando sus principales características, y funcionamiento, partiendo de un modelo genérico aplicable a cualquier fuente de energía que interviene en la red eléctrica.

Se nombraron distintos tipos de sistemas empleados actualmente en la conversión de energía eólica y de energía solar indicando su topología, funcionalidad y características.

\section{BIBIOGRAFÍA}

1. Blaabjerg F, Chen Z, Teodorescu R, Iov F, editors. Power Electronics in Wind Turbine Systems. Power Electronics and Motion Control Conference, 2006 IPEMC 2006 CES/IEEE 5th International; 2006 14-16 Aug. 2006.

2. Blaabjerg F, Iov F, Chen Z, Ma K, editors. Power electronics and controls for wind turbine systems. Energy Conference and Exhibition (EnergyCon), 2010 IEEE International; 2010 1822 Dec. 2010.

3. Iov F, Ciobotaru M, Blaabjerg F, editors. Power electronics control of wind energy in distributed power systems. Optimization of Electrical and Electronic Equipment, 2008 OPTIM 2008 11th International Conference on; 2008 22-24 May 2008.

4. Hansen LH, Madsen PH, Blaabjerg F, Christensen HC, Lindhard U, Eskildsen K, editors. Generators and power electronics technology for wind turbines. Industrial Electronics Society, 2001 IECON '01 The 27th Annual Conference of the IEEE; 20012001.

5. Hyong Sik K, Lu DDC, editors. Review on wind turbine generators and power electronic converters with the grid-connection issues. Universities Power Engineering Conference (AUPEC), 2010 20th Australasian; 2010 5-8 Dec. 2010.

6. Zhe C, Guerrero JM, Blaabjerg F. A Review of the State of the Art of Power Electronics for Wind Turbines. Power Electronics, IEEE Transactions on. 2009;24(8):1859-75.

7. Huang AQ, Bhattacharya S, Baran M, Bin C, Chong H, editors. Active Power Management of Electric Power System Using Emerging Power Electronics Technology. Power Engineering Society General Meeting, 2007 IEEE; 2007 24-28 June 2007.

8. Biczel P, Jasinski A, Lachecki J, editors. Power Electronic Devices in Modern Power Systems. EUROCON, 2007 The International Conference on \&\#34;Computer as a Tool\&\#34;; 2007 9-12 Sept. 2007.

9. Shinde SM, Patil KD, Khairnar SS, Gandhare WZ, editors. The Role of Power Electronics in Renewable Energy Systems Research and Development. Emerging Trends in Engineering and Technology (ICETET), 2009 2nd International Conference on; 2009 16-18 Dec. 2009. 